\title{
Hidden nonmacrorealism: Reviving the Leggett-Garg inequality with stochastic operations
}

\author{
Huan-Yu Ku $\odot,{ }^{1,2, *}$ Hao-Cheng Weng $\odot,{ }^{3,}{ }^{*}$ Yen-An Shih $\odot,{ }^{3}$ Po-Chen Kuo $\odot,{ }^{1,2}$ Neill Lambert $\odot,{ }^{2}$ Franco Nori $\odot, 2,4,5$ \\ Chih-Sung Chuu $\left(\mathbb{0},{ }^{3, \dagger}\right.$ and Yueh-Nan Chen ${ }^{1,2, \$}$ \\ ${ }^{1}$ Department of Physics and Center for Quantum Frontiers of Research and Technology (QFort), \\ National Cheng Kung University, Tainan 701, Taiwan \\ ${ }^{2}$ Theoretical Quantum Physics Laboratory, RIKEN Cluster for Pioneering Research, Wako-shi, Saitama 351-0198, Japan \\ ${ }^{3}$ Department of Physics, National Tsing Hua University, Hsinchu 30013, Taiwan and Center for Quantum Technology, Hsinchu 30013, Taiwan \\ ${ }^{4}$ RIKEN Center for Quantum Computing, Wako, Saitama 351-0198, Japan \\ ${ }^{5}$ Department of Physics, The University of Michigan, Ann Arbor, Michigan 48109-1040, USA
}

(Received 18 May 2021; accepted 12 October 2021; published 29 October 2021)

\begin{abstract}
The Leggett-Garg inequality (LGI) distinguishes nonmacrorealistic channels from macrorealistic ones by constraining the experimental outcomes of the underlying system. In this work, we propose a class of channels which, initially, cannot violate the LGI (in the form of the temporal Bell inequality) but can violate it after the application of stochastic pre- and post-operations (SPPOs). As a proof-of-principle experiment, we demonstrate the stochastic pre- and post-operations in an amplitude-damping channel with photonic qubits. We denote the above phenomenon as hidden nonmacrorealistic channels. We also discuss the relationship between these hidden nonmacrorealistic channels [in terms of the temporal Clauser-Horne-Shimony-Holt (CHSH) inequality] and the strongly nonlocality-breaking channel, which breaks the hidden spatial CHSH nonlocality for arbitrary states. In general, if the channel satisfies hidden nonmacrorealism, it is not a strongly CHSH nonlocality-breaking channel.
\end{abstract}

DOI: 10.1103/PhysRevResearch.3.043083

\section{INTRODUCTION}

The location of the boundary [1] between classical macroscopic systems, which have definite or pre-existing physical properties, and microscopic quantum objects, obeying the uncertainty principle, is still under debate. In the seminal work [2], Leggett and Garg provided a quantitative way to broach this problem with an inequality which distinguishes genuine quantum superpositions from macroscopic systems with definite (macrorealistic) observables. Violating the Leggett-Garg inequality (LGI) implies that the dynamics of the system is nonmacrorealistic, which means the experimental outcomes violate either macrorealism per se or noninvasive measurability (or both) [3]. Without loss of generality, the dynamics of the system, which is used to construct the two-time correlation function for computing the LGI, can always be described by quantum channels. Therefore, when there is no ambiguity, throughout this work, we will use the terminology of quantum channels instead of the dynamical process of the system.

Based on the definition of macrorealism, variations and refinements of the original LGI can be derived, including the

\footnotetext{
${ }^{*}$ These authors contributed equally to this work.

$\dagger$ cschuu@phys.nthu.edu.tw

‡yuehnan@mail.ncku.edu.tw
}

Published by the American Physical Society under the terms of the Creative Commons Attribution 4.0 International license. Further distribution of this work must maintain attribution to the author(s) and the published article's title, journal citation, and DOI. quantum witness (or no-signaling-in-time condition) [4-8], temporal Bell inequalities [9,10], transport-based inequalities [11-13], higher dimensional LGIs [14-16], multitime correlations [17-19], and the partial exclusion of false violations arising from clumsy (classically invasive) measurement [20-24]. In addition, the range of such tests can be presented as a hierarchy of temporal correlations [25-28]. Furthermore, many significant experiments have been implemented in different systems, such as photons [29-32], superconducting qubits [21,24,33-36], and atoms [37,38]. Recently, temporal quantum correlations (including LGI) have found applications for quantum information and communication problems, like witnessing the dimension of a system [39-41], the certification of quantum memory [42-47], quantum clock [48], enhancing the quantumness of a system with nonMarkovianity [49,50], self-testing the quantum measurement [51], and verifying the quantumness of a channel [52-56].

In this work, we propose a class of quantum channels that alone cannot violate the temporal Bell inequality. However, after applying stochastic pre- and post-operations (SPPOs), this class of channels can violate the temporal Bell inequality. We denote such channels as "hidden nonmacrorealistic channels", akin to "hidden nonlocality" in the context of the spatial Bell inequality [57-59], in which a local state can be activated to become nonlocal by stochastic local operations and classical communication [60-64]. In comparing these two similar scenarios, we find an additional implication of our result for strongly Clauser-Horne-Shimony-Holt (CHSH) nonlocality-breaking channels, which break the hidden $\mathrm{CHSH}$ nonlocality for arbitrary states [65]. We show that when the channel satisfies hidden nonmacrorealism, it is not a strongly 


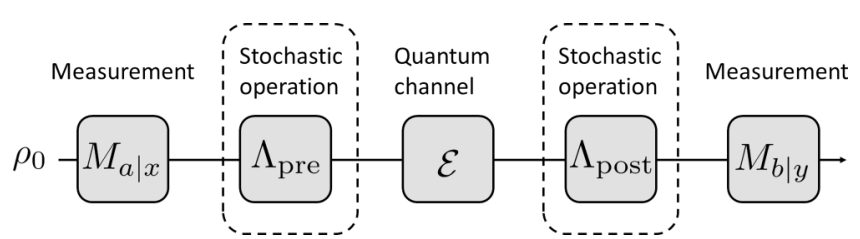

FIG. 1. Schematic diagram. Without the process in the dashedline boxes, the schematic diagram represents the standard nonmacrorealism test. Hidden nonmacrorealism can be observed by introducing the stochastic operations in the dashed-boundary boxes.

CHSH nonlocality-breaking channel. Therefore, the LGI can be applied in the certification of the quantumness in a quantum network.

This work is organized as follows. In Sec. II, we introduce the formalism and basic concepts related to macrorealism and strongly CHSH nonlocality-breaking channel. In Sec. III, we introduce the concept of hidden nonmacrorealism under the temporal CHSH scenario and its relation to the strongly CHSH nonlocality-breaking channel. In Sec. IV, we experimentally demonstrate the hidden nonmacrorealism in an amplitude-damping channel. Finally, in Sec. V, we present the conclusion and outlook of our work.

\section{PRELIMINARY NOTIONS}

\section{A. Temporal Clauser-Horne-Shimony-Holt inequality}

In this section, we briefly recall the Leggett-Garg inequality (LGI) in the form of the temporal Bell inequality $[3,9,10]$. We consider a system undergoing sequential measurements with classical inputs $x(y)$ and outcomes $a(b)$ acting at time $t_{0}$ $\left(t_{1}\right)$. A schematic diagram of this process is shown in Fig. 1. If the dynamics of the system obeys macrorealism per se (the system is always in a macroscopic state), and noninvasive measurability (measurements only reveal the physical property of the state without disturbance [2,3]), the observed probability distribution for the outcomes $\{p(a, b \mid x, y)\}$ must be macrorealistic. That is,

$$
p(a, b \mid x, y) \stackrel{\mathrm{MS}}{=} \sum_{\lambda} p(\lambda) p(a \mid x, \lambda) p(b \mid y, \lambda)
$$

where $\lambda$ is the hidden parameter which determines causally all physical properties including the probability distributions $p(a \mid x, \lambda)$ and $p(b \mid y, \lambda)$.

In contrast, employing quantum theory, the observed probability distribution can be formulated by Born's rule, namely,

$$
p(a, b \mid x, y) \stackrel{\mathrm{QM}}{=} \operatorname{Tr}\left[M_{b \mid y} \mathcal{E}\left(M_{a \mid x} \rho_{0} M_{a \mid x}\right)\right], \forall a, b, x, y .
$$

Here, $\rho_{0}$ is the initial state, $\mathcal{E}$ is a quantum channel describing the dynamics between time $t_{0}$ and $t_{1}$, and $\left\{M_{a \mid x}\right\}\left(\left\{M_{b \mid y}\right\}\right)$ is the von Neumann measurement set. It has been shown that there exist statistics (generated by quantum theory) not admitting Eq. (1) $[3,9,10,66,67]$. Thus, we denote such a correlation in time as nonmacrorealism.
The assumption of noninvasive measurability can be recast as the so-called no-signalling-in-time condition $[4-8,68]$,

$$
\sum_{a} p(a, b \mid x, y)=\sum_{a} p\left(a, b \mid x^{\prime}, y\right) \forall x \neq x^{\prime} .
$$

The above formula indicates that the choice of the measurement at time $t_{0}$ does not "disturb" the observing probability distribution at time $t_{1}$. To satisfy Eq. (3) for an arbitrary measurement set $\left\{M_{a \mid x}\right\}$, we can consider the initial state $\rho_{0}$ to be the maximally mixed state because the unnormalized post-measurement state for von Neumann measurement can be described by $M_{a \mid x} \rho_{0} M_{a \mid x}$.

To demonstrate our main result, it is convenient to introduce the simplest temporal Bell inequality with the index sets $x, y \in\{1,2\}$ and $a, b \in\{ \pm 1\}$. The temporal Clauser-HorneShimony-Holt (CHSH) inequality is [10]

$$
B_{\mathrm{T}-\mathrm{CHSH}}=C_{1,1}+C_{2,1}+C_{1,2}-C_{2,2} \leqslant 2,
$$

where 2 is the macrorealistic bound, and $C_{x, y}=\sum_{a, b} a$. $b p(a, b \mid x, y)$ is the two-time correlation function. Here, the indices " $a$ " and " $b$ " represent the measurement outcomes of Alice and Bob with measurement bases labeled by $x$ and $y$ at different times $t_{0}$ and $t_{1}$, respectively. If the temporal CHSH inequality is not violated, the correlation is regarded as $\mathrm{CHSH}$ macrorealistic. We note that there is no difference between the temporal CHSH and the standard CHSH inequality unless one a priori knows how to generate the probability distributions.

\section{B. Strongly Clauser-Horne-Shimony-Holt nonlocality-breaking channel}

Before showing our main result, we briefly recall the concept of the (strongly) CHSH nonlocality-breaking channel $[65,69]$. A bipartite state $\rho_{\mathrm{AB}}$ is said to be CHSH nonlocal if it violates the CHSH inequality, which has the same form of Eq. (4). Otherwise, we say the state is CHSH local. In addition, we say that a state is hidden CHSH nonlocal if a CHSH local state can become nonlocal after applying stochastic local operations with classical communication. Here, we use the local filtering operations, denoted by $\Lambda_{\mathrm{A}}$ and $\Lambda_{\mathrm{B}}$, to implement the stochastic local operations with classical communication [61-63]. A bipartite state after local filters can be described by

$$
\rho_{\mathrm{AB}}^{\prime}=\left(K_{\mathrm{A}} \otimes K_{\mathrm{B}}\right)\left(\rho_{\mathrm{AB}}\right)\left(K_{\mathrm{A}}^{\dagger} \otimes K_{\mathrm{B}}^{\dagger}\right) / N,
$$

where $K_{\mathrm{A}}\left(K_{\mathrm{B}}\right)$ is the Kraus representation of the local filtering operation, and $N=\operatorname{Tr}\left[\left(K_{\mathrm{A}} \otimes K_{\mathrm{B}}\right)\left(\rho_{\mathrm{AB}}\right)\left(K_{\mathrm{A}}^{\dagger} \otimes K_{\mathrm{B}}^{\dagger}\right)\right]$ is the normalization constant. In general, a local filtering operation is a complete-positive (CP) trace-nonincreasing map with only one Kraus operator. Therefore, we can define another Kraus operator, $K_{2}=\sqrt{\mathbb{1}-K_{1}^{\dagger} K_{1}}$, such that $K_{1}^{\dagger} K_{1}+K_{2}^{\dagger} K_{2}=$ $\mathbb{1}$ forms a quantum channel. In addition, since there are only two Kraus operators in the above quantum channel, the system through the local filter $\left(K_{1}\right)$ can be regarded as a successful operation. On the other hand, the system after $K_{2}$ is denoted as a fail operation. In this case, $N$ represents the success probability. Now, if a correlation, obtained after the channel $\mathcal{E}$ acting on the subsystem, always satisfies the CHSH inequality for arbitrary measurements and bipartite states, the channel is $\mathrm{CHSH}$ nonlocality-breaking. More specifically, a $\mathrm{CHSH}$ 
nonlocality-breaking channel generates the correlation

$$
p(a, b \mid x, y)=\operatorname{Tr}\left[M_{a \mid x} \otimes M_{b \mid x}(\mathbb{1} \otimes \mathcal{E}) \rho_{\mathrm{AB}}\right],
$$

which always satisfies the $\mathrm{CHSH}$ inequality with arbitrary measurement sets $\left\{M_{a \mid x}\right\}\left(\left\{M_{b \mid y}\right\}\right)$, and state $\rho_{\mathrm{AB}}$. Note that the measurement $M_{a \mid x}\left(M_{b \mid y}\right)$ is implemented on one of the spatially separated states. Furthermore, if the evolved state $(\mathbb{1} \otimes \mathcal{E}) \rho_{\mathrm{AB}}$ does not show hidden $\mathrm{CHSH}$ nonlocality for any input state and any choice of measurements, the channel can be further denoted as a strongly $\mathrm{CHSH}$ nonlocalitybreaking channel. It has been shown [65] that the strongly CHSH nonlocality-breaking channel can be certified by testing whether the Choi state of the channel, defined as $\rho_{\mathrm{CJ}}=$ $(\mathbb{1} \otimes \mathcal{E})\left|\Phi_{+}\right\rangle\left\langle\Phi_{+}\right|$with $\left|\Phi_{+}\right\rangle=1 / \sqrt{d} \sum_{i}|i\rangle \otimes|i\rangle$, shows the hidden $\mathrm{CHSH}$ nonlocality. Note that the Choi state can be defined not only for the quantum channel but also for CP maps [70-72].

\section{HIDDEN NONMACROREALISM}

We now consider a more general protocol by which we can "activate" nonmacrorealism from apparently macrorealistic channels by introducing stochastic pre- and post-operations (SPPOs). The schematic diagram of the process is shown in Fig. 1. More precisely, given a quantum channel $\mathcal{E}$, that obeys Eq. (1) for arbitrary measurements $\left\{M_{a \mid x}\right\}$ and $\left\{M_{b \mid y}\right\}$, nonmacrorealism can be observed after applying the SPPOs described by the pre and post filters $\Lambda_{\text {pre }}$ and $\Lambda_{\text {post }}$. The probability distribution of the outcomes from the whole process including measurements, SPPOs, and channel can now be formulated as

$$
p(a, b \mid x, y)=\operatorname{Tr}\left[M_{b \mid y}\left(\frac{\Lambda_{\text {post }} \circ \mathcal{E} \circ \Lambda_{\text {pre }}\left(M_{a \mid x}\right)}{d \times N(a \mid x)}\right)\right],
$$

where the normalization constants are

$$
N(a \mid x)=\operatorname{Tr}\left[\Lambda_{\text {post }} \circ \mathcal{E} \circ \Lambda_{\text {pre }}\left(M_{a \mid x}\right)\right] / d .
$$

Here, the initial input with dimension $d$ has already been inserted. Note that, in general, the success probability depends on the initial state. However, since we consider the maximally mixed state as the input, the postmeasurement states correspond to eigenstates of the von Neumann measurement operators. Therefore, the success probability depends on the measurement set. Similar to the concept in hidden $\mathrm{CHSH}$ nonlocality, the CHSH macrorealistic channel, which can be activated to a CHSH nonmacrorealistic one by SPPOs, is denoted as hidden $\mathrm{CHSH}$ nonmacrorealistic channel.

Physically, $\Lambda$ can be experimentally implemented by weak measurements [73], or a filtering process [60,61,74-77]. In the next section, we will explicitly show how filtering operations can activate a hidden $\mathrm{CHSH}$ nonmacrorealistic channel. We also note that weak measurements have been used to experimentally demonstrate standard nonmacrorealism in different physical systems, including photonic systems [29], superconducting circuits [34], and atom transport systems [38].

Recently, it has been shown that a channel which is not a CHSH nonlocality-breaking channel can be witnessed by violating the temporal CHSH inequality [56]. In other words, if the temporal CHSH inequality can be violated, the channel is not CHSH nonlocality-breaking. Here, we further show how temporal quantum correlations can be used to distinguish the strongly $\mathrm{CHSH}$ nonlocality-breaking channels from their counterparts [65].

We consider the Choi representation of the channel with pre and post filters, namely $\rho_{\mathrm{CJ}}:=\left(\mathbb{1} \otimes \Lambda_{\text {post }} \circ \mathcal{E} \circ\right.$ $\left.\Lambda_{\text {pre }}\right)\left|\Phi_{+}\right\rangle\left\langle\Phi_{+}\right.$, the output state under a general physical map (CP map is up to renormalization) $\Lambda(X)=\operatorname{Tr}_{\text {in }}\left[\mathbb{1} \otimes X^{\top} \rho_{\mathrm{CJ}}\right]$ [78], and the property of the maximally entangled state $(\mathbb{1} \otimes$ $X)\left|\Phi_{+}\right\rangle=\left(X^{\top} \otimes \mathbb{1}\right)\left|\Phi_{+}\right\rangle$. Here, $X$ represents any operator and $\mathrm{T}$ denotes the transpose. To compare with the strongly $\mathrm{CHSH}$ nonlocality-breaking channel, we further assume that the normalization constants are uniform $N(a \mid x)=N \forall a, x$ [cf. Eq. (5)]. In other words, the success probability is independent of the choice of the measurement set $\left\{M_{a \mid x}\right\}$ (see also Ref. [44] which discusses the same issue in the LGI). Inserting the above into Eq. (7), we can see that testing hidden $\mathrm{CHSH}$ nonmacrorealistic channels is the same as certifying whether the Choi state of the channel $\mathcal{E}$ shows hidden $\mathrm{CHSH}$ nonlocality (cf., Sec. II [65]). Equivalently, if there is hidden $\mathrm{CHSH}$ nonmacrorealism, the corresponding channel is not strongly $\mathrm{CHSH}$ nonlocality-breaking. Thus, the temporal Bell inequality can be used to certify the quantumness of a quantum channel in a quantum network.

\section{EXPERIMENTAL DEMONSTRATION}

As a proof-of-principle demonstration, we consider the hidden $\mathrm{CHSH}$ nonmacrorealism in an amplitude-damping channel with photonic qubits. The amplitude damping is commonly used to describe the energy dissipation or spontaneous emission. An amplitude-damping channel $\mathcal{E}(\rho)$ can be decomposed into

$$
\mathcal{E}(\rho)=E_{1} \rho E_{1}^{\dagger}+E_{2} \rho E_{2}^{\dagger}
$$

with

$$
\begin{gathered}
E_{1}=\sqrt{1-v}|1\rangle\langle 1|+| 0\rangle\langle 0|, \\
E_{2}=\sqrt{v}|0\rangle\langle 1|,
\end{gathered}
$$

corresponding to the decay of the population in $|1\rangle$ and the quantum jump from $|1\rangle$ to $|0\rangle$, respectively, and $v \in$ $[0,1]$ being the visibility. At high visibility, the qubit in the amplitude-damping channel tends to be in the state $|0\rangle$ and exhibits CHSH macrorealism $\left(B_{\mathrm{T}-\mathrm{CHSH}} \leqslant 2\right)$ for $v \geqslant v_{\text {th }}=$ 0.5 . However, as demonstrated by our experiment, the $\mathrm{CHSH}$ nonmacrorealism $\left(B_{\mathrm{T}-\mathrm{CHSH}}>2\right)$ can be observed for $v>v_{\text {th }}$ if the following SPPO (filtering processes) are exploited,

$$
\begin{aligned}
& K_{\text {pre }}=|0\rangle\langle 0|+\sqrt{1-D}| 1\rangle\langle 1|, \\
& K_{\text {post }}=\sqrt{1-D}|0\rangle\langle 0|+| 1\rangle\langle 1|,
\end{aligned}
$$

where $D \in[0,1]$ is the power loss of state $|0\rangle$.

\section{A. Experimental setup}

The schematic of our experimental setup is illustrated in Fig. 2. The type-II quasi-phase-matched spontaneous parametric down-conversion (SPDC) is realized in a periodically poled lithium niobate waveguide pumped by a 780-nm CW laser (ECDL system). The co-propagating 1560-nm photon 


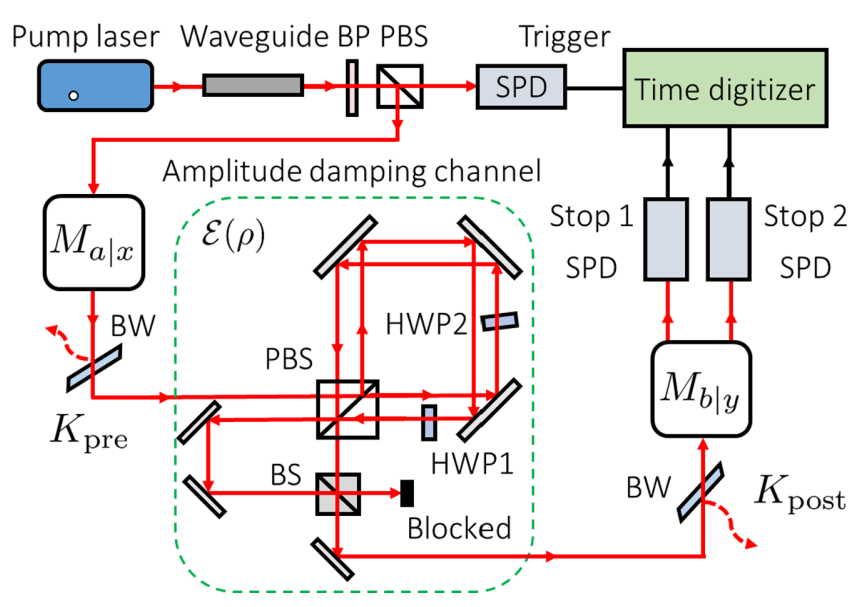

FIG. 2. Experimental setup. BP: Band-pass filter; SPD: singlephoton detection modules. BW: Brewster window plate. The time digitizer is triggered by the horizontally polarized photons. The coincidence counts are recorded once the measurements $M_{b \mid y}$ lead to clicks at Stop 1 or Stop 2.

pairs are spatially separated with a polarizing beam splitter (PBS). This allows us to herald a single photon in the signal channel by detecting an idler photon. To test the temporal CHSH inequality, we send a maximally mixed state into the amplitude-damping channel at $t_{0}$ by preparing the qubit in an ensemble of the eigenstates of $\hat{\sigma}_{x}$ and $\hat{\sigma}_{y}$ with equal probability [79]. In addition, we use the von Neumann measurement sets $\left\{M_{a \mid x}\right\}=\left\{\hat{\sigma}_{x}, \hat{\sigma}_{y}\right\}$ at time $t_{0}$ and $\left\{M_{b \mid y}\right\}=$ $\left\{\left(\hat{\sigma}_{x}+\hat{\sigma}_{y}\right) / \sqrt{2},\left(\hat{\sigma}_{x}-\hat{\sigma}_{y}\right) / \sqrt{2}\right\}$ at time $t_{1}$.

The amplitude-damping channel is implemented in a Sagnac-like interferometer $[73,80]$ with polarization encoding, $|0\rangle=|H\rangle$ and $|1\rangle=|V\rangle$. The $|H\rangle$ and $|V\rangle$ components of the qubit are first separated by a PBS. The $|V\rangle$ component is then passed though a half-wave plate (HWP1), with its fast axis rotated at $0<\theta<\pi / 2(\sin 2 \theta=\sqrt{v})$, and converted probabilistically to the $|H\rangle$ component after the PBS. To compensate the additional phase induced by HWP1, another half-wave plate (HWP2) tilted at an angle is inserted in the interferometer arm of the $|H\rangle$ component. After leaving the interferometer, the $|H\rangle$ component is temporally delayed by traveling a longer optical path than that by the $|V\rangle$ component before the two components are recombined incoherently on a nonpolarizing beam-splitter (BS). By doing so, an initial state like

$$
\rho=(\alpha|H\rangle+\beta|V\rangle)\left(\alpha^{*}\langle H|+\beta^{*}\langle V|\right)
$$

is altered to

$$
\begin{aligned}
\mathcal{E}(\rho)= & (\alpha|H\rangle+\beta \cos 2 \theta|V\rangle)\left(\alpha^{*}\langle H|+\beta^{*} \cos 2 \theta\langle V|\right) \\
& +\beta^{2} \sin ^{2} 2 \theta|H\rangle\langle H|,
\end{aligned}
$$

in the amplitude-damping channel. Essential to our demonstration is the $K_{\text {pre }}$ and $K_{\text {post }}$ of the SPPOs. These operators are implemented by the glass windows placed at the Brewster angles. By adjusting the principle axes of the windows, we can filter out the $|V\rangle$ or $|H\rangle$ component, which corresponds to the actions $\Lambda_{\text {pre }}$ and $\Lambda_{\text {post }}$ in Fig. 2. In our experiment, $D$ is approximately 0.45 for both $K_{\text {pre }}$ and $K_{\text {post }}$.

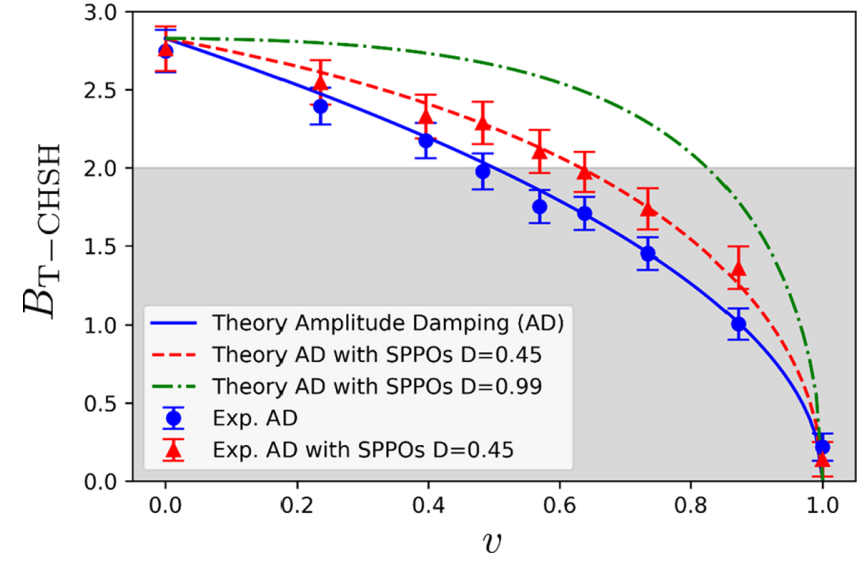

FIG. 3. Observation of the hidden CHSH nonmacrorealism in an amplitude-damping channel with visibility $v$. The grey shaded area at the bottom represent the macrorealistic regime. Without applying the SPPOs (blue dots with solid curves being the theoretical prediction), the temporal CHSH inequality is violated $B_{\mathrm{T}-\mathrm{CHSH}}>2$ up to $v=0.48$. With the SPPOs (red triangles with dashed curves), the violations are observed up to $v=0.64$. The green dot-dashed curve represents the theoretical prediction with loss $D \approx 1$ (where $D=0.99$ ). The error bars take into account the finite accuracy of the waveplate's angle $\left( \pm 1^{\circ}\right)$, imperfect Brewster windows $(D \pm$ $2 \%$ ), misaligned incident polarization $\left( \pm 1^{\circ}\right)$, and nonideal visibility (96-98\%) of the Sagnac interferometer.

\section{B. Experimental results}

The hidden $\mathrm{CHSH}$ nonmacrorealism is experimentally probed by the violation of the temporal $\mathrm{CHSH}$ inequality Eq. (4). Figure 3 shows the measured $B_{\mathrm{T}-\mathrm{CHSH}}$ versus the visibility $v=\sin ^{2} 2 \theta$. Without the SPPOs (circular dots), we observe the CHSH nonmacrorealism up to $v=0.48$, which is in good agreement with the theory (solid curve). The slight deviation between our observation and theory likely results from the finite accuracy of the waveplate's angle, nonideal Brewster windows, misaligned incident polarization, and imperfect visibility of the Sagnac interferometer. After applying the SPPOs (triangular dots), the violation of the temporal $\mathrm{CHSH}$ inequality can be observed up to $v_{\max }=$ $0.64>v_{\text {th }}$, thus demonstrating the hidden CHSH nonmacrorealism in the region of $0.5<v \leqslant 0.64$. We note that the range of the observable hidden $\mathrm{CHSH}$ nonmacrorealism is only limited by the power loss $D$ chosen for the SPPOs in Eq. (12). For example, the hidden CHSH nonmacrorealism can be observed up to $v_{\max }=0.83$ if $D \approx 1$, which can be achieved by using more glass windows at the Brewster angles. We also note that when $v>0.5$, the channel is CHSH nonlocality-breaking [69]. Therefore, our experimental observation implies that the amplitude-damping channel is not a strongly CHSH-nonlocality-breaking channel [65] for $0.5<v \leqslant 0.64$.

\section{DISCUSSION}

In this work, we have proposed and demonstrated hidden nonmacrorealism. More specifically, we have shown how macrorealistic channels can be "activated" to nonmacrorealistic channels by using the stochastic pre- and post-operations 
(SPPOs) both theoretically and experimentally. In other words, our proposal provides a heuristic way to partially mitigate certain types of temporal quantum correlations against decoherence. In addition, we have shown how strongly $\mathrm{CHSH}$ nonlocality-breaking channels are related to the hidden $\mathrm{CHSH}$ nonmacrorealistic channels. In general, when the system is hidden $\mathrm{CHSH}$ nonmacrorealistic, it is not a strongly $\mathrm{CHSH}$ nonlocality-breaking channel.

This work also suggests some open problems. How can one determine the set of hidden nonmacrorealistic channels? Is it enough to test whether the Choi state of the channel shows the hidden nonmacrorealistic channels? As we have shown in our experiment, certain consequences of decoherence can be suppressed by applying SPPOs. In fact, similar ideas can be generalized and applied to different problems, e.g., reviving quantum interference [81] and quantum teleportation power [82].

\section{ACKNOWLEDGMENTS}

The authors acknowledge fruitful discussions with S.L. Chen, C.-Y. Cheng, J.-Y. Li, and G. N. M. Tabia. H.-Y.Ku acknowledges the support of the Ministry of Science and Technology, Taiwan (Grant No. MOST 110-2811-M006-546). N.L. acknowledges partial support from JST PRESTO through Grant No. JPMJPR18GC. This work is supported partially by the National Center for Theoretical Sciences and Ministry of Science and Technology, Taiwan, Grants No. MOST 110-2123-M-006-001 and No. MOST 109-2627-M-006-004, and the Army Research Office (under Grant No. W911NF-19-1-0081). H.-C.W., Y.-A.Sh., and C.-S.C. acknowledge the support of the Ministry of Science and Technology, Taiwan (107-2112-M-007-004-MY3). F.N. is supported in part by Nippon Telegraph and Telephone Corporation (NTT) Research, the Japan Science and Technology Agency (JST) [via the Quantum Leap Flagship Program (Q-LEAP) program, the Moonshot R\&D Grant No. JPMJMS2061, and the Centers of Research Excellence in Science and Technology (CREST) Grant No. JPMJCR1676], the Japan Society for the Promotion of Science (JSPS) [via the Grants-in-Aid for Scientific Research (KAKENHI) Grant No. JP20H00134 and the JSPS-RFBR Grant No. JPJSBP120194828], the Army Research Office (ARO) (Grant No. W911NF-18-1-0358), the Asian Office of Aerospace Research and Development (AOARD) (via Grant No. FA2386-20-1-4069), and the Foundational Questions Institute Fund (FQXi) via Grant No. FQXi-IAF19-06.
[1] E. Schrödinger, Discussion of probability relations between separated systems, Proc. Cambr. Philos. Soc. 31, 555 (1935).

[2] A. J. Leggett and A. Garg, Quantum Mechanics Versus Macroscopic Realism: Is the Flux There when Nobody Looks? Phys. Rev. Lett. 54, 857 (1985).

[3] C. Emary, N. Lambert, and F. Nori, Leggett-Garg inequalities, Rep. Prog. Phys. 77, 016001 (2014).

[4] C.-M. Li, N. Lambert, Y.-N. Chen, G.-Y. Chen, and F. Nori, Witnessing quantum coherence: From solid-state to biological systems, Sci. Rep. 2, 885 (2012).

[5] J. Kofler and C. Brukner, Condition for macroscopic realism beyond the Leggett-Garg inequalities, Phys. Rev. A 87, 052115 (2013).

[6] L. Clemente and J. Kofler, No Fine Theorem for Macrorealism: Limitations of the Leggett-Garg Inequality, Phys. Rev. Lett. 116, 150401 (2016).

[7] J. J. Halliwell, Leggett-Garg inequalities and no-signaling in time: A quasiprobability approach, Phys. Rev. A 93, 022123 (2016).

[8] J. J. Halliwell, Comparing conditions for macrorealism: Leggett-Garg inequalities versus no-signaling in time, Phys. Rev. A 96, 012121 (2017).

[9] C. Brukner, S. Taylor, S. Cheung, and V. Vedral, Quantum entanglement in time, arXiv:quant-ph/0402127.

[10] T. Fritz, Quantum correlations in the temporal Clauser-HorneShimony-Holt (CHSH) scenario, New J. Phys. 12, 083055 (2010).

[11] N. Lambert, C. Emary, Y.-N. Chen, and F. Nori, Distinguishing Quantum and Classical Transport Through Nanostructures, Phys. Rev. Lett. 105, 176801 (2010).
[12] N. Lambert, Y.-N. Chen, and F. Nori, Unified single-photon and single-electron counting statistics: From cavity qed to electron transport, Phys. Rev. A 82, 063840 (2010).

[13] C. Emary, N. Lambert, and F. Nori, Leggett-Garg inequality in electron interferometers, Phys. Rev. B 86, 235447 (2012).

[14] C. Budroni and C. Emary, Temporal Quantum Correlations and Leggett-Garg Inequalities in Multilevel Systems, Phys. Rev. Lett. 113, 050401 (2014).

[15] S. V. Moreira, A. Keller, T. Coudreau, and P. Milman, Modeling Leggett-Garg-inequality violation, Phys. Rev. A 92, 062132 (2015).

[16] N. Lambert, K. Debnath, A. F. Kockum, G. C. Knee, W. J. Munro, and F. Nori, Leggett-Garg inequality violations with a large ensemble of qubits, Phys. Rev. A 94, 012105 (2016).

[17] M. Ringbauer, F. Costa, M. E. Goggin, A. G. White, and A. Fedrizzi, Multi-time quantum correlations with no spatial analog, npj Quantum Inf. 4, 37 (2018).

[18] R. Uola, G. Vitagliano, and C. Budroni, Leggett-Garg macrorealism and the quantum nondisturbance conditions, Phys. Rev. A 100, 042117 (2019).

[19] G. D. Berk, A. J. P. Garner, B. Yadin, K. Modi, and F. A. Pollock, Resource theories of multi-time processes: A window into quantum non-Markovianity, Quantum 5, 435 (2021).

[20] M. M. Wilde and A. Mizel, Addressing the clumsiness loophole in a Leggett-Garg test of macrorealism, Found. Phys. 42, 256 (2011).

[21] G. C. Knee, K. Kakuyanagi, M.-C. Yeh, Y. Matsuzaki, H. Toida, H. Yamaguchi, S. Saito, A. J. Leggett, and W. J. Munro, A strict experimental test of macroscopic realism in a superconducting flux qubit, Nat. Commun. 7, 13253 (2016). 
[22] C. Emary, Ambiguous measurements, signaling, and violations of Leggett-Garg inequalities, Phys. Rev. A 96, 042102 (2017).

[23] S. V. Moreira and M. T. Cunha, Quantifying quantum invasiveness, Phys. Rev. A 99, 022124 (2019).

[24] H.-Y. Ku, N. Lambert, F.-J. Chan, C. Emary, Y.-N. Chen, and F. Nori, Experimental test of non-macrorealistic cat states in the cloud, npj Quantum Inf. 6, 98 (2020).

[25] Y.-N. Chen, C.-M. Li, N. Lambert, S.-L. Chen, Y. Ota, G.-Y. Chen, and F. Nori, Temporal steering inequality, Phys. Rev. A 89, 032112 (2014).

[26] C.-M. Li, Y.-N. Chen, N. Lambert, C.-Y. Chiu, and F. Nori, Certifying single-system steering for quantum-information processing, Phys. Rev. A 92, 062310 (2015).

[27] H.-Y. Ku, S.-L. Chen, N. Lambert, Y.-N. Chen, and F. Nori, Hierarchy in temporal quantum correlations, Phys. Rev. A 98, 022104 (2018).

[28] R. Uola, F. Lever, O. Gühne, and J.-P. Pellonpää, Unified picture for spatial, temporal, and channel steering, Phys. Rev. A 97, 032301 (2018).

[29] M. E. Goggin, M. P. Almeida, M. Barbieri, B. P. Lanyon, J. L. O'Brien, A. G. White, and G. J. Pryde, Violation of the Leggett-Garg inequality with weak measurements of photons, Proc. Natl. Acad. Sci. USA 108, 1256 (2011).

[30] J. Dressel, C. J. Broadbent, J. C. Howell, and A. N. Jordan, Experimental Violation of Two-Party Leggett-Garg Inequalities with Semiweak Measurements, Phys. Rev. Lett. 106, 040402 (2011).

[31] Z.-Q. Zhou, S. F. Huelga, C.-F. Li, and G.-C. Guo, Experimental Detection of Quantum Coherent Evolution Through the Violation of Leggett-Garg-Type Inequalities, Phys. Rev. Lett. 115, 113002 (2015).

[32] K. Wang, M. Xu, L. Xiao, and P. Xue, Experimental violations of Leggett-Garg inequalities up to the algebraic maximum for a photonic qubit, Phys. Rev. A 102, 022214 (2020).

[33] A. Palacios-Laloy, F. Mallet, F. Nguyen, P. Bertet, D. Vion, D. Esteve, and A. N. Korotkov, Experimental violation of a Bell's inequality in time with weak measurement, Nat. Phys. 6, 442 (2010).

[34] G. C. Knee, S. Simmons, E. M. Gauger, J. J. L. Morton, H. Riemann, N. V. Abrosimov, P. Becker, H.-J. Pohl, K. M. Itoh, M. L. Thewalt, G. A. D. Briggs, and S. C. Benjamin, Violation of a Leggett-Garg inequality with ideal non-invasive measurements, Nat. Commun. 3, 606 (2012).

[35] T. C. White, J. Y. Mutus, J. Dressel, J. Kelly, R. Barends, E. Jeffrey, D. Sank, A. Megrant, B. Campbell, Y. Chen, Z. Chen, B. Chiaro, A. Dunsworth, I.-C. Hoi, C. Neill, P. J. J. O’Malley, P. Roushan, A. Vainsencher, J. Wenner, A. N. Korotkov et al., Preserving entanglement during weak measurement demonstrated with a violation of the Bell-Leggett-Garg inequality, npj Quantum Inf. 2, 15022 (2016).

[36] E. Huffman and A. Mizel, Violation of noninvasive macrorealism by a superconducting qubit: Implementation of a Leggett-Garg test that addresses the clumsiness loophole, Phys. Rev. A 95, 032131 (2017).

[37] C. Budroni, G. Vitagliano, G. Colangelo, R. J. Sewell, O. Gühne, G. Tóth, and M. W. Mitchell, Quantum Nondemolition Measurement Enables Macroscopic Leggett-Garg Tests, Phys. Rev. Lett. 115, 200403 (2015).

[38] C. Robens, W. Alt, D. Meschede, C. Emary, and A. Alberti, Ideal Negative Measurements in Quantum Walks Disprove The- ories Based on Classical Trajectories, Phys. Rev. X 5, 011003 (2015).

[39] G. Schild and C. Emary, Maximum violations of the quantumwitness equality, Phys. Rev. A 92, 032101 (2015).

[40] J. Hoffmann, C. Spee, O. Gühne, and C. Budroni, Structure of temporal correlations of a qubit, New J. Phys. 20, 102001 (2018).

[41] C. Spee, H. Siebeneich, T. F. Gloger, P. Kaufmann, M. Johanning, M. Kleinmann, C. Wunderlich, and O. Gühne, Genuine temporal correlations can certify the quantum dimension, New J. Phys. 22, 023028 (2020).

[42] D. Rosset, F. Buscemi, and Y.-C. Liang, Resource Theory of Quantum Memories and Their Faithful Verification with Minimal Assumptions, Phys. Rev. X 8, 021033 (2018).

[43] C. Budroni, G. Fagundes, and M. Kleinmann, Memory cost of temporal correlations, New J. Phys. 21, 093018 (2019).

[44] R. Uola, T. Kraft, and A. A. Abbott, Quantification of quantum dynamics with input-output games, Phys. Rev. A 101, 052306 (2020).

[45] S. Langenfeld, O. Morin, M. Körber, and G. Rempe, A networkready random-access qubits memory, npj Quantum Inf. 6, 86 (2020).

[46] L. B. Vieira and C. Budroni, Temporal correlations in the simplest measurement sequences, arXiv:2104.02467.

[47] X. Yuan, Y. Liu, Q. Zhao, B. Regula, J. Thompson, and M. Gu, Universal and operational benchmarking of quantum memories, npj Quantum Inf. 7, 108 (2021).

[48] C. Budroni, G. Vitagliano, and M. P. Woods, Ticking-clock performance enhanced by nonclassical temporal correlations, Phys. Rev. Research 3, 033051 (2021).

[49] K.-D. Wu, Z. Hou, G.-Y. Xiang, C.-F. Li, G.-C. Guo, D. Dong, and F. Nori, Detecting non-Markovianity via quantified coherence: Theory and experiments, npj Quantum Inf. 6, 55 (2020).

[50] S. Milz, D. Egloff, P. Taranto, T. Theurer, M. B. Plenio, A. Smirne, and S. F. Huelga, When is a Non-Markovian Quantum Process Classical? Phys. Rev. X 10, 041049 (2020).

[51] A. G. Maity, S. Mal, C. Jebarathinam, and A. S. Majumdar, Self-testing of binary Pauli measurements requiring neither entanglement nor any dimensional restriction, Phys. Rev. A 103, 062604 (2021).

[52] M. F. Pusey, Verifying the quantumness of a channel with an untrusted device, J. Opt. Soc. Am. B 32, A56 (2015).

[53] Y. Wang, I. W. Primaatmaja, E. Lavie, A. Varvitsiotis, and C. C. W. Lim, Characterising the correlations of prepare-andmeasure quantum networks, npj Quantum Inf. 5, 17 (2019).

[54] J.-D. Lin, W.-Y. Lin, H.-Y. Ku, N. Lambert, Y.-N. Chen, and F. Nori, Witnessing quantum scrambling with steering, Phys. Rev. A 104, 022614 (2021).

[55] S.-H. Chen, H. Lu, Q.-C. Sun, Q. Zhang, Y.-A. Chen, and C.-M. Li, Discriminating quantum correlations with networking quantum teleportation, Phys. Rev. Research 2, 013043 (2020).

[56] H.-Y. Ku, J. Kadlec, A. Černoch, M. Túlio Quintino, W. Zhou, K. Lemr, N. Lambert, A. Miranowicz, S.-L. Chen, F. Nori, and Y.-N. Chen, Detecting quantum non-breaking channels without entanglement, arXiv:2106.15784.

[57] J. S. Bell, On the Einstein Podolsky Rosen paradox, Physics 1, 195 (1964).

[58] J. F. Clauser, M. A. Horne, A. Shimony, and R. A. Holt, Proposed Experiment to Test Local Hidden-Variable Theories, Phys. Rev. Lett. 23, 880 (1969). 
[59] N. Brunner, D. Cavalcanti, S. Pironio, V. Scarani, and S. Wehner, Bell nonlocality, Rev. Mod. Phys. 86, 419 (2014).

[60] S. Popescu, Bell's Inequalities and Density Matrices: Revealing "Hidden" Nonlocality, Phys. Rev. Lett. 74, 2619 (1995).

[61] N. Gisin, Hidden quantum nonlocality revealed by local filters, Phys. Lett. A 210, 151 (1996).

[62] L. Masanes, Y.-C. Liang, and A. C. Doherty, All Bipartite Entangled States Display Some Hidden Nonlocality, Phys. Rev. Lett. 100, 090403 (2008).

[63] F. Hirsch, M. T. Quintino, J. Bowles, and N. Brunner, Genuine Hidden Quantum Nonlocality, Phys. Rev. Lett. 111, 160402 (2013).

[64] Y. Wang, J. Li, X.-R. Wang, T.-J. Liu, and Q. Wang, Experimental demonstration of hidden nonlocality with local filters, Opt. Express 28, 13638 (2020).

[65] R. Pal and S. Ghosh, Non-locality breaking qubit channels: The case for CHSH inequality, J. Phys. A: Math. Theor. 48, 155302 (2015).

[66] G. Waldherr, P. Neumann, S. F. Huelga, F. Jelezko, and J. Wrachtrup, Violation of a Temporal Bell Inequality for Single Spins in a Diamond Defect Center, Phys. Rev. Lett. 107, 090401 (2011).

[67] S.-S. Majidy, J. J. Halliwell, and R. Laflamme, Detecting violations of macrorealism when the Leggett-Garg inequalities are satisfied, Phys. Rev. A 103, 062212 (2021).

[68] J. J. Halliwell, Leggett-Garg tests of macrorealism: Checks for noninvasiveness and generalizations to higher-order correlators, Phys. Rev. A 99, 022119 (2019).

[69] Y. Zhang, R. A. Bravo, V. O. Lorenz, and E. Chitambar, Channel activation of CHSH nonlocality, New J. Phys. 22, 043003 (2020).

[70] J. Bavaresco, M. Araújo, Č. Brukner, and M. T. Quintino, Semi-device-independent certification of indefinite causal order, Quantum 3, 176 (2019).
[71] A. A. Abbott, J. Wechs, D. Horsman, M. Mhalla, and C. Branciard, Communication through coherent control of quantum channels, Quantum 4, 333 (2020).

[72] C. Giarmatzi and F. Costa, Witnessing quantum memory in nonMarkovian processes, Quantum 5, 440 (2021).

[73] Y.-S. Kim, J.-C. Lee, O. Kwon, and Y.-H. Kim, Protecting entanglement from decoherence using weak measurement and quantum measurement reversal, Nat. Phys. 8, 117 (2011).

[74] R. Horodecki, P. Horodecki, M. Horodecki, and K. Horodecki, Quantum entanglement, Rev. Mod. Phys. 81, 865 (2009).

[75] M. Horodecki, P. Horodecki, and R. Horodecki, Mixed-State Entanglement and Distillation: Is There a "Bound" Entanglement in Nature? Phys. Rev. Lett. 80, 5239 (1998).

[76] P. G. Kwiat, S. Barraza-Lopez, A. Stefanov, and N. Gisin, Experimental entanglement distillation and 'hidden' non-locality, Nature (London) 409, 1014 (2001).

[77] H.-Y. Ku, C.-Y. Hsieh, S.-L. Chen, C. Budroni, and Y.-N. Chen, Quantifying measurement incompatibility with universal distillation of quantum steering (unpublished).

[78] G. Chiribella, G. M. D'Ariano, and P. Perinotti, Theoretical framework for quantum networks, Phys. Rev. A 80, 022339 (2009).

[79] K. Bartkiewicz, A. Černoch, K. Lemr, A. Miranowicz, and F. Nori, Experimental temporal quantum steering, Sci. Rep. 6, 38076 (2016).

[80] M. P. Almeida, F. de Melo, M. Hor-Meyll, A. Salles, S. P. Walborn, P. H. S. Ribeiro, and L. Davidovich, Environmentinduced sudden death of entanglement, Science 316, 579 (2007).

[81] C.-H. Wu, C.-K. Liu, Y.-C. Chen, and C.-S. Chuu, Revival of Quantum Interference by Modulating the Biphotons, Phys. Rev. Lett. 123, 143601 (2019).

[82] J.-Y. Li, X.-X. Fang, T. Zhang, G. N. M. Tabia, H. Lu, and Y.C. Liang, Activating hidden teleportation power: Theory and experiment, Phys. Rev. Research 3, 023045 (2021). 